\title{
Deep Vein Thrombosis and Subsequent Pulmonary Embolism in A Patient Recovered from COVID-19
}

\section{Elena Kordzaya $^{1}$ | Oleg Dukhin ${ }^{1}$ | Polina Golovina ${ }^{1}$ | Darya Vorobyeva ${ }^{2}$ | Elena Maryukhnich ${ }^{3}$ | Polina Savvinova $^{*}$ | Elena Vasilieva ${ }^{5}$ | Alexander Shpektor ${ }^{6}$}

\section{*Correspondence: Polina Savvinova}

Address: ${ }^{1}$ Cardiology Fellow. Laboratory of Atherothrombosis, Cardiology Department, State University of Medicine and Dentistry, Moscow, Russia; ${ }^{2}$ Research assistant. Laboratory of Atherothrombosis, State University of Medicine and Dentistry, Moscow, Russia; ${ }^{3}$ Researcher. Laboratory of Atherothrombosis, State University of Medicine and Dentistry, Moscow, Russia; ${ }^{4}$ Assistant Professor. Clinical City Hospital named after I.V. Davydovsky, Moscow Department of Healthcare, Moscow, Russia; ${ }^{5}$ Professor. Laboratory of Atherothrombosis, Head. State University of Medicine and Dentistry, Moscow, Russia; ${ }^{6}$ Professor. Cardiology Department, Head, State University of Medicine and Dentistry, Moscow, Russia e-mail $\bowtie$ : psavvinova@yandex.ru

Received: 01 October 2020; Accepted: 06 October 2020

Copyright: (C) 2020 Kordzaya E. This is an open-access article distributed under the terms of the Creative Commons Attribution License, which permits unrestricted use, distribution, and reproduction in any medium, provided that the original work is properly cited.

\section{ABSTRACT}

A 45-year-old female patient with a previous SARS-CoV2 pneumonia suddenly presented with pain, swelling and cyanosis of the left leg, and severe dyspnea. Extensive deep vein thrombosis of the left leg and bilateral pulmonary embolism were diagnosed. The patient received standard treatment according to ESC 2019 Guidelines on Acute Pulmonary Embolism (Konstantinides et al., 2019). A study of the patient's cytokine profile was carried out, according to which an increase in several pro-inflammatory cytokines was detected. After a 3-week follow-up, partial recanalization of the deep veins of the left leg was revealed.

Keywords: COVID-19, Pulmonary Embolism, Cytokines, Thrombosis

\section{Introduction}

Despite active study of both the fundamental and the clinical features of the novel coronavirus disease (COVID-19) that the world has faced in 2020, there is currently a lack of data on the long-term prognosis of patients who have contracted this infection. A high prevalence of thrombotic complications in patients with COVID-19 has recently been described (Tang et al., 2020a). However, many questions regarding thromboembolism risk stratification, the choice of anticoagulant and its dose, and the duration of anticoagulant therapy in this group of patients are yet to be clarified. Although there is some consensus on these issues, further randomized clinical trials are needed (Spyropoulos et al., 2020a; Thachil et al., 2020). 
We present a case of ileofemoral thrombosis and pulmonary embolism in a patient with a predisposition for thromboembolic events with previous novel coronavirus pneumonia.

\section{Timeline}

\begin{tabular}{|c|c|}
\hline Time & Event \\
\hline 2 weeks before admission & Covid-19 along with bilateral pneumonia treatment in hospita \\
\hline 1,5 weeks before admission & Sudden onset of exertional dyspnea \\
\hline 1 day before admission & Left leg cyanosis along with pain and swelling \\
\hline Admission date & Severe dyspnea \\
\hline Primary inspection & $\begin{array}{l}\text { Signs of segmentary pulmonary embolism on CT - pulmonary } \\
\text { angiography along with left leg occlusive deep vein } \\
\text { thrombosis }\end{array}$ \\
\hline Day 1 & Anticoagulation therapy with UFH was initiated \\
\hline Day 5 & Switch to NOACs treatment \\
\hline 3 weeks after release & Signs of left leg deep vein recanalization \\
\hline
\end{tabular}

\section{Case Presentation}

\section{Anamnesis Morbi}

A 45-year old woman was admitted to our hospital because of pronounced dyspnea, left leg cyanosis, and swelling. It was found that two weeks before then she had been treated in a hospital for viral pneumonia caused by SARS-CoV2. During her stay in the hospital (21/04/2020), the patient experienced exertional dyspnea. In blood tests, the level of D-dimer was increased to $1830 \mathrm{ng} / \mathrm{ml}$. However, anticoagulant therapy was not carried out, because of hemorrhoidal bleeding. On $30 / 04 / 2020$, the patient was discharged from the hospital after receiving double negative results on the PCR test for SARS-CoV-2.

01/05/2020 a sharp pain in the left inguinal region with further irradiation of pain on the inner surface of the thigh and lower leg was observed, followed by swelling and cyanosis of the left leg. The next day, the patient developed an attack of severe resting dyspnea, accompanied by decreases in blood pressure to 70/50 mm Hg and in SpO2 down to 91\%. The patient was admitted to our hospital. It was noteworthy that the patient had taken combined oral contraceptives for a year and, moreover, she had used intravaginal gestagen-estrogen-containing rings because of endometriosis-associated dysmenorrhea.

Upon admission, the patient's respiratory rate was 20/min, Sp02 was 95\%, and blood pressure 
was 110/70 mm Hg; therefore thrombolysis was not performed.

Investigations

Laboratory studies revealed leukocytosis $(18.5 * 109 / \mathrm{L})$ and increased level of C-reactive protein (92.7 mg / L) and D-dimer (> $1500 \mathrm{ng} / \mathrm{ml}$ ). The ECG was unremarkable. A contrast CT-scan revealed defects of the pulmonary artery contrasting at the segmental and subsegmental levels of both lungs as well as in the lower lobes of both lungs, together with areas of lung tissue opacity with involvement of the pulmonary parenchyma in the amount of $25 \%$, which met the criteria for the severity of the lesion (CT-1) (Fig. 1). There were no echocardiographic signs of right heart failure. Ultrasonography of the lower-extremity veins revealed occlusive deep vein thrombosis of the left leg (Fig. 2).

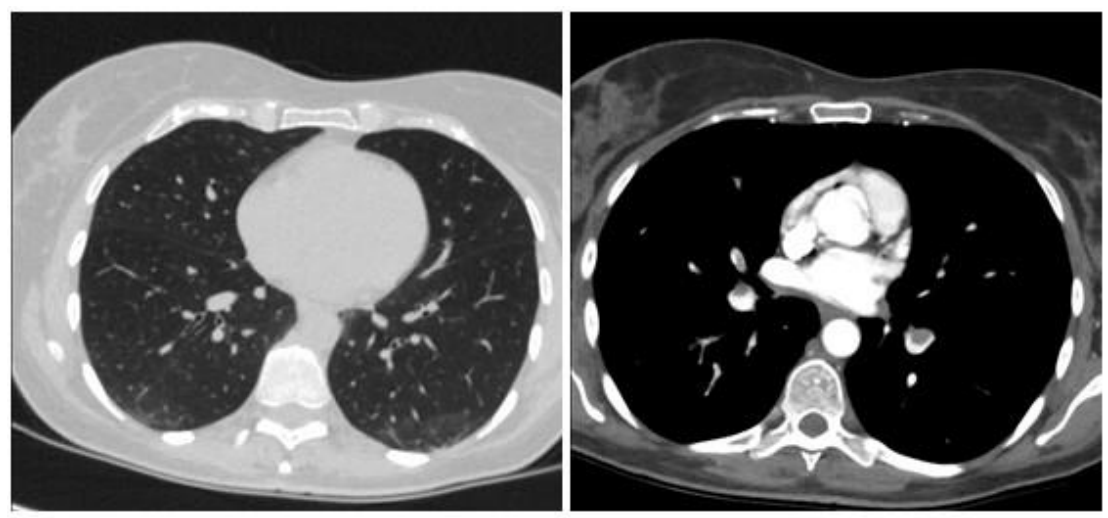

Figure 1: Contrast CT-scan

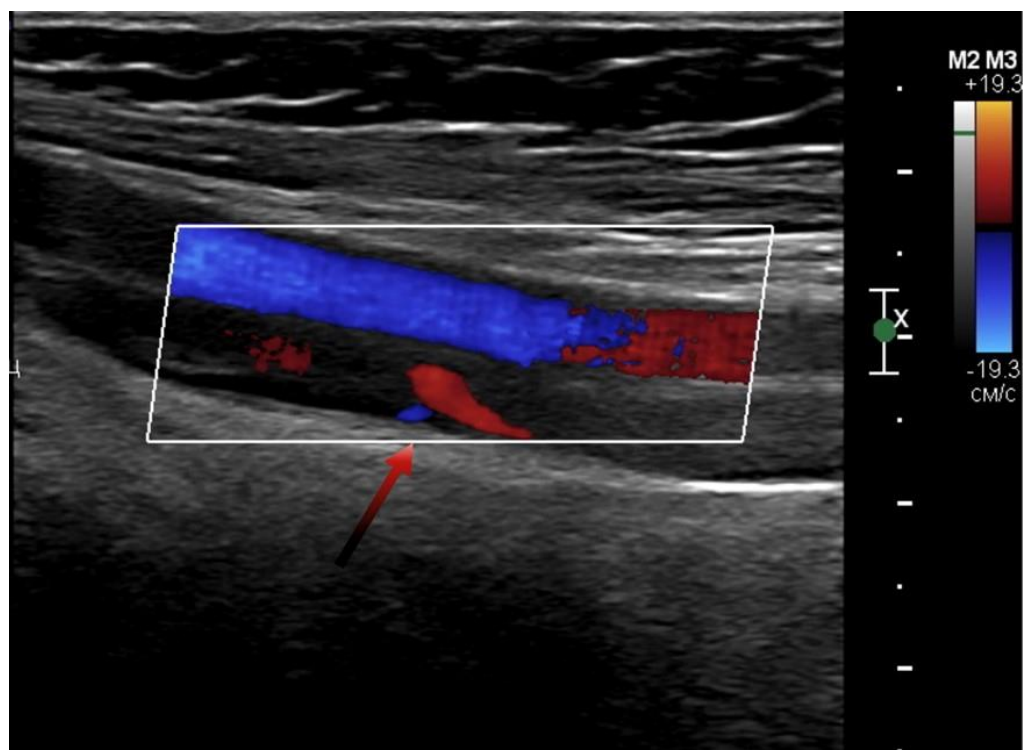

Figure 2: Ultrasonography of the lower extremity

In order to access endothelial function, we performed a flowmediated dilatation test. The test showed a normal value $(13,1 \%)$. 


\section{Management}

In the acute period of pulmonary embolism, the patient was treated with UFH under the control of the APTT. During treatment, the patient's condition remained stable, and echocardiographic parameters did not worsen in dynamics (PAP $35 \mathrm{~mm} \mathrm{Hg}$ ). Subsequently, the patient was switched to rivaroxaban (15 mg b.i.d. for 3 weeks), and a prolongation of anticoagulation for 6 months (rivaroxaban 20 mg q.d.) was recommended. Removal of the intravaginal ring was not carried out because of the high risk of developing menometrorrhagia due to ongoing anticoagulant therapy.

A follow-up examination was performed after 3 weeks, and partial recanalization of the deep veins of the left lower limb was noted. After discharge, the patient tolerated physical activity well without recurrence of dyspnea. An echocardiographic examination revealed no negative dynamics compared with anamnestic data.

\section{Cytokine Study}

We measured several proinflammatory cytokine concentration in the patient's plasma. According to our results, concentrations of IFN-y, IL-13, IL-6 (see full list of elevated cytokines in Suppl. Table 2) were more than 3 SD higher than the average concentrations of these cytokines in healthy controls.

\section{Discussion}

Viral infections are known to induce endothelial disfunction, inflammatory state, and hypoxemia which can lead to subsequent thrombosis (Gupta et al., 2019; Phillippe, 2017). COVID-19 is associated with hypercoagulation with a high risk of thromboembolic complications (Tang et al., 2020). According to our data, activity of COVID-19-induced coagulopathy correlates with disease severity, while generalized endothelial dysfunction was not observed (Kalinskaya et al., 2020). In this clinical case, the patient also had a normal FMD-test.

However, VTE is described mainly in the acute period of infection, and there are no robust data on the timing of anticoagulation after discharge from the hospital.

In this case report, in a patient with a procoagulant state (use of combined contraceptive vaginal ring,) tending to bleeding (hemorrhoidal and uterine), SARS-CoV2 infection provoked ileofemoral thrombosis and pulmonary embolism two weeks after the acute period of infection, despite normal endothelial function.

Several inflammatory cytokines have been previously shown to be risk factors for deep vein 
thrombosis and pulmonary embolism (Christiansen et al., 2006; Halici et al., 2014).

Recently, plasma levels of numerous cytokines have been shown to be increased in COVID-19infected patients (Rothan and Byrareddy, 2020; Spiezia et al., 2020) as well as in patients with pulmonary embolism. It has been shown that some of the cytokines we observed (IFN-y, IL-1 $\beta$, IL-6) may be associated with hypercoagulation and subsequent thromboembolic events (Bester and Pretorius, 2016). The mechanisms of their action include enhancing neutrophil extracellular trap (NET) formation, inducing platelet activation, and promoting vein wall inflammation) (Bertin et al., 2019; Nosaka et al., 2011; Zhang et al., 2020).

All these facts together correlate with our findings from this case: higher levels of proinflammatory cytokines involved in COVID-19 may also take part in hypercoagulation and deep vein thrombosis.

Thus, anticoagulation is the cornerstone of COVID-19 management (Bikdeli et al., 2020; Thachil et al., 2020). Various protocols of anticoagulant therapy have been developed. Current guidelines recommend the use of unfractioned heparin and low molecular weight heparins (Atallah et al., 2020; Connors and Levy, 2020; Rico-Mesa et al., 2020). At the same time, the issue of prolonged anticoagulant therapy and its duration remains controversial. The routine use of long-term anticoagulation after COVID-19 infection seems to be inappropriate because of the high risk of bleeding complications. However, there are certain high-risk patient groups that require long-term anticoagulation. The use of previously developed protocols for prolonged thromboprophylaxis was proposed in patients with a high risk of thromboembolic complications (active cancer, history of DVT, etc.), as well as a more than two fold increased level of D-dimer (Cohen et al., 2014; Spyropoulos et al., 2020). The use of direct oral anticoagulants may also be considered (Cohen et al., 2016), but there is currently no evidence comparing the effectiveness of oral and parenteral prolonged anticoagulation regimens for COVID-19 patients. Further research efforts are needed to develop criteria for prolonged anticoagulation and its duration.

\section{Conclusion}

In this case we observed a thrombotic complication in a patient recovered from SARS-CoV2induced pneumonia. In addition to factors predisposing to hypercoagulation, a hyperproduction of several proinflammatory cytokines (IFN-y, IL-1 $\beta$, IL-6) induced by SARS-CoV-2 was also revealed. This case report demonstrates the necessity of careful hemostasis study in patients with COVID-19 and the development of anticoagulation algorithms after discharge. 
Declaration of Competing Interest: The authors declare that the research was conducted in the absence of any commercial or financial relationships that could be construed as a potential conflict of interest.

Ethical Approval: Ethical approval to report this case was obtained from the local ethics committee.

Funding: Moscow Ministry of Healthcare.

Informed Consent: Written informed consent was obtained from legally authorized representatives for anonymized patient information to be published in this article.

Author Contribution: EK, OD, PG contributed to the literature search, data interpretation and writing of the manuscript. DV, EM provided a cytokine study, contributed to the literature search, data interpretation and writing of the manuscript. PS, AS, EV critically reviewed the manuscript.

Acknowledgements: We thank Barry Alpher for correcting the English style of the manuscript.

\section{References}

Atallah B, Mallah SI, AlMahmeed W. Anticoagulation in COVID-19. Eur Hear Journal Cardiovasc Pharmacother 2020; 6: 260-261.

Bertin FR, Rys RN, Mathieu C, Laurance S, Lemarié CA, Blostein MD. Natural killer cells induce neutrophil extracellular trap formation in venous thrombosis. J Thromb Haemost 2019; 17: 403-414.

Bester J and Pretorius E. Effects of IL-1ß, IL-6 and IL-8 on erythrocytes, platelets and clot viscoelasticity. Sci Rep 2016; 6: $1-10$.

Bikdeli B, Madhavan MV, Jimenez D, Chuich T, Dreyfus I, Driggin E, Nigoghossian C, Ageno W, Madjid M, Guo Y, Tang LV. COVID-19 and Thrombotic or Thromboembolic Disease: Implications for Prevention, Antithrombotic Therapy, and Follow-Up: JACC State-of-the-Art Review. J Am Coll Cardiol 2020; 75: 2950-2973.

Christiansen SC, Næss IA, Cannegieter SC, Hammerstrøm J, Rosendaal FR, Reitsma PH. Inflammatory Cytokines as Risk Factors for a First Venous Thrombosis: A Prospective Population-Based Study. PLoS Med 2006; 3: e334.

Cohen AT, Harrington RA, Goldhaber SZ, Hull RD, Wiens BL, Gold A, Hernandez AF, Gibson CM. Extended thromboprophylaxis with betrixaban in acutely ill medical patients. N Engl J Med 2016; 375: 534-544.

Cohen AT, Spiro TE, Spyropoulos AC, Desanctis YH, Homering M, Büller HR, Haskell L, Hu D, Hull R, Mebazaa A, Merli G. D-dimer as a predictor of venous thromboembolism in acutely ill, hospitalized patients: A subanalysis of the randomized controlled MAGELLAN trial. J Thromb Haemost 2014; 12: 479-487.

Connors JM and Levy JH. COVID-19 and its implications for thrombosis and anticoagulation. Blood 2020.

Gupta N, Zhao YY, Evans CE. The stimulation of thrombosis by hypoxia. Thromb Res 2019; 181: 77-83.

Halici B, Ulasli SS, Günay E, Nural S, Sen S, Akar O, Celik S, Unlu M. Assessment of inflammatory biomarkers and oxidative stress in pulmonary thromboembolism: Follow-up results. Inflammation 2014; 37: 1186-1190. 
Kalinskaya A, Dukhin O, Molodtsov I, Maltseva A, Sokorev D, Elizarova A, Sapozhnikova O, Glebova K, Stonogina D, Shakhidzhanov S, Nikonov E. Dynamics of coagulopathy in patients with different COVID-19 severity. MedRxiv 2020: 2020.07.02.20145284.

Konstantinides SV, Meyer G, Becattini C, Bueno H, Geersing GJ, Harjola VP, Huisman MV, Humbert M, Jennings CS, Jiménez D, Kucher N. 2019 ESC Guidelines for the diagnosis and management of acute pulmonary embolism developed in collaboration with the European Respiratory Society (ERS). Eur Respir J 2019; 54: 1901647.

Nosaka M, Ishida Y, Kimura A, Kuninaka Y, Inui M, Mukaida N, Kondo T. Absence of IFN- $\gamma$ accelerates thrombus resolution through enhanced MMP-9 and VEGF expression in mice. J Clin Invest 2011; 121: 2911-2920.

Phillippe HM. Overview of venous thromboembolism. Am J Manag Care 2017; 23: S376-S382.

Rico-Mesa JS, Rosas D, Ahmadian-Tehrani A, White A, Anderson AS, Chilton R. The Role of Anticoagulation in COVID-19Induced Hypercoagulability. Curr Cardiol Rep 2020; 22.

Rothan HA and Byrareddy SN. The epidemiology and pathogenesis of coronavirus disease (COVID-19) outbreak. J Autoimmun 2020; 109: 102433.

Spiezia L, Boscolo A, Poletto F, Cerruti L, Tiberio I, Campello E, Navalesi P, Simioni P. COVID-19-Related Severe Hypercoagulability in Patients Admitted to Intensive Care Unit for Acute Respiratory Failure. Thromb Haemost 2020; 120: 9981000.

Spyropoulos AC, Levy JH, Ageno W, Connors JM, Hunt BJ, Iba T, Levi M, Samama CM, Thachil J, Giannis D, Douketis JD. Scientific and Standardization Committee Communication: Clinical Guidance on the Diagnosis, Prevention and Treatment of Venous Thromboembolism in Hospitalized Patients with COVID-19. J Thromb Haemost 2020a: jth.14929.

Spyropoulos AC, Lipardi C, Xu J, Peluso C, Spiro TE, De Sanctis Y, Barnathan ES, Raskob GE. Modified IMPROVE VTE Risk Score and Elevated D-Dimer Identify a High Venous Thromboembolism Risk in Acutely Ill Medical Population for Extended Thromboprophylaxis. TH Open 2020b; 04: e59-e65.

Tang N, Li D, Wang X, Sun Z. Abnormal coagulation parameters are associated with poor prognosis in patients with novel coronavirus pneumonia. J Thromb Haemost 2020a; 18: 844-847.

Thachil J, Tang N, Gando S, Falanga A, Levi M, Clark C, Iba T, Cattaneo M. Type and dose of heparin in COVID-19. J Thromb Haemost 2020.

Zhang Y, Zhang Z, Wei R, Miao X, Sun S, Liang G, Chu C, Zhao L, Zhu X, Guo Q, Wang B. IL (Interleukin)-6 Contributes to Deep Vein Thrombosis and Is Negatively Regulated by miR-338-5p. Arterioscler Thromb Vasc Biol 2020; 40: 323-334. 